\title{
Antimicrobials Induced hyponatremia in elderly patients
}

\begin{abstract}
Low serum sodium concentration is not an uncommon frequent electrolyte disorder in the elderly population because of multiple risk factors contributing to increased antidiuretic hormone, the frequent prescription of drugs associated with hyponatremia, and also because of other mechanisms, such as the "tea and toast" syndrome. Herein, we focus on antibiotics' rare contribution to hyponatremia in elderly. Hyponatremia in elderly subjects is mainly induced by medications (more frequently thiazides and antidepressants), the syndrome of inappropriate antidiuretic hormone secretion (SIAD) or endocrinopathies; however, hyponatremia is multifactorial in a significant proportion of patients. Special attention is needed in the elderly population to exclude endocrinopathies as a cause of hyponatremia before establishing the diagnosis of SIAD, which then requires a stepped diagnostic approach to reveal its underlying cause. The treatment of hyponatremia depends on the type of hyponatremia and special attention is also needed to correct serum sodium levels at the appropriate rate, especially in chronic hyponatremia, in order to avoid the osmotic demyelination syndrome. In conclusion, both the evaluation and the treatment of hyponatremia constitute many challenges in the elderly population.
\end{abstract}

Keywords: antibiotics in aging patients, hyponatremia, acute kidney injury, intestinal nephritis
Volume 7 Issue 5 - 2019

\author{
Abdullah Elhosiny,' Ahmed Akl, ${ }^{1,2,3}$ Nezar \\ Bahabri ${ }^{1,2,3}$ \\ 'Dr Soliman Fakeeh Hospital, Saudi Arabia \\ ${ }^{2}$ Fakeeh College of Medical Sciences, Saudi Arabia \\ ${ }^{3}$ Urology \& Nephrology Center, Mansoura University, Egypt
}

Correspondence: Ahmed Akl, Associate Professor, Consultant of Nephrology \& Transplantation, Urology \& Nephrology Center, Mansoura University, Egypt,

Email aiak12001@yahoo.com

Received: September 14,2019 | Published: September 27, 2019

\section{Introduction}

\section{Nitrofurantoin}

Nitrofurantoin is effective on microorganisms in a very low inhibitory concentration reaching $32 \mu \mathrm{g} / \mathrm{ml}$ or less. Ninety percent reach circulation unchanged, and forty percent excreted in urine. Its blood concentration reaches a peak after an oral dose of 100 $\mathrm{mg}$, it has poor tissue penetration, and is rapidly metabolized by the liver. However, its high concentration in urine only one quart excreted unchanged in the urine. Based on data from experimental studies, the urinary excretion is a mixture of glomerular filtration and tubular secretion. In fact, high urine $\mathrm{pH}$ inhibits the overall tubular re-absorption and maintain nitrofurantoin concentration in urine. ${ }^{1}$ In addition, nitrofurantoin inhibitory activity increases dramatically when $\mathrm{pH}$ rises above 6 . Its bacteriostatic activity is against wide variety of organisms at a low concentration reaching $32 \mathrm{ug} / \mathrm{ml}$, it is bactericide in a concentration above $100 \mathrm{ug} / \mathrm{ml} .^{2} \mathrm{E}$. coli rare to acquired resistance to nitrofurantoin, it may be due to inhibition of nitrofurantoin reductase either chromosomal or plasmid-mediated. ${ }^{3}$ It is not preferred to be utilized in advanced chronic kidney disease starting from stage $3 \mathrm{~B}$ due to two facts: first, low sub-therapeutic concentration in urine, and second, due to critical accumulation of metabolites in blood leading to numerous side effects. ${ }^{4-5}$ Those severe side effects are more prone in elderly patients. Thus, it is not recommended to give it for the elderly population as per the American Geriatrics Society 2019 Updated Beers Criteria for Potentially Inappropriate Medication Use in Older Adults. Side effects can be serious in case of glutathione instability, so it is contra-indicated in conditions with immature enzyme stability as in $3^{\text {rd }}$ trimester pregnant women, infants up to one month, and patients with glucose-6-phosphate dehydrogenase deficiency because of risk of intravascular hemolysis resulting in anemia. In elderly patient, it induced hyponatremia.

\section{Co-trimoxazole}

Co-trimoxazole is a combination (5:1) of Trimethoprim and sulfamethoxazole. This combination has synergistic bactericidal effect by inhibition of bacterial biosynthesis of nucleic acids and proteins necessary for their survival through inhibition of the production of titrahydrofolic acid by trimethoprim and synthesis of the dihydrofolic acid by sulfamethoxazole. Its side effects are mainly on the gastro-intestinal tract, various allergic reaction types on the skin, on the blood and bone marrow suppression, and even fulminant hepatitis has been described. Co-trimoxazole can induce hyponatremia, but the mechanism is unknown. Trimethoprim is similar in the biochemical structure to potassium sparing diuretics, so it inhibits sodium reabsorption of sodium in exchange with potassium at the distal tubule ending with hyponatremia associated with hyperkalemia. ${ }^{6-8} \mathrm{~A}$ retrospective study was performed to identify if electrolytes derangement related to co-trimoxazole dose or not. Overall, electrolyte derangement was more prone with high doses, but still present with low doses especially in those with low GFR.

Many reports of hyponatremia post co-trimoxazole been published, one HIV patient suffered pneumocystis carinii received co-trimoxazole $20 \mathrm{mg} / \mathrm{kg}$, four days later his serum sodium dropped from 137 to 121 $\mathrm{mml} / 1$ once co-trimoxazole discontinued his sodium level normalized in a couple of days. ${ }^{9}$ In another clinical scenario, a 75-year-old woman with recurrent urinary tract infections, and a normal serum sodium of $136 \mathrm{mmol} / \mathrm{L}$ was found to have hyponatremia once she received cotrimoxazole combined with hydrochlorothiazide, and hyponatremia recovers if she receives hydrochlorothiazide alone or co-trimoxazole alone. ${ }^{10}$ The explanation referred to the dual block of the sodium transporters by the combined action of the two medications on renal tubules especially in elderly patients with expected impaired tubular activity. It was reported that hyponatremia was induced by co- 
trimoxazole in a synergistic effect with anti-psychiatric medications. Seventy-nine-year-old woman with previously normal serum sodium $149 \mathrm{mmol} / \mathrm{l}$ was admitted to hospital with serum sodium $119 \mathrm{mmol} / \mathrm{l}$, after receiving citalopram and co-trimoxazole prior to admission by 7 days. ${ }^{11}$ An eighty-six-year-old man suffered hyponatremia within days from starting co-trimoxazole for urinary tract infection. ${ }^{12}$ In both patients, there was a similar observed hyponatremia with the initiation of co-trimoxazole and improvement after discontinuation of therapy, especially in elderly patients with high scale of Naranjo ADRPS nine out of thirteen. ${ }^{13}$

\section{Fluoroquinolones}

\section{Ciprofloxacin}

Ciprofloxacin has been reported to cause serious hyponatremia. The hypothesized mechanism of action is due to ciprofloxacin crossing the blood-brain barrier, resulting in stimulation of the $\gamma$-aminobutyric acid and N-methyl-D-aspartate receptors, leading to more release of antidiuretic hormone. ${ }^{14}$

\section{Levofloxacin}

There was two case reports of associated hyponatremia with the use of levofloxacin. However, the evidence is not strong as hyponatremia in such cases was multifactorial, and may be related to nasopharyngeal cancer. ${ }^{15}$

\section{Moxifloxacin}

Is the fourth generation of the fluoroquinolones, prescribed for the empirical coverage of pneumococcal and anaerobic infections. ${ }^{16}$ Its main side effects include toxicity on the gastrointestinal and central nervous system (CNS), less common, ECG changes, hypoglycemia, transaminitis, phototoxicity, tendon and joint disorders, and hypersensitivity reactions. ${ }^{17}$ CNS toxicities range from mild as headache and nausea, to rare severe CNS toxicities as seizures in patients who are susceptible to and have other risk factors for seizures. ${ }^{18}$ Rarely, fluoroquinolones was reported to have caused hyponatremia. Two case reports of moxifloxacin to cause hyponatremia, the first case report moxifloxacin use was combined with thiazide diuretics which may be an added factor. ${ }^{19}$ The second case report of moxifloxacin associated hyponatremia after its utilization in a COPD patient for 5 days. $^{20}$

\section{Linezolid}

Oxazolidinone antibiotic against Gram-positive bacteria, however, thrombocytopenia is a major adverse effect of linezolid; hyponatremia is a common association after administration. In a retrospective observational cohort study, higher frequency of hyponatremia (sodium less than $134 \mathrm{meq} / \mathrm{l})$ in the group of patients received linezolid and potassium, sparing diuretics, compared to other groups $(\mathrm{p}=0.016) .{ }^{21} \mathrm{~A}$ case of severe hyponatremia associated with linezolid induced SAIDH was reported. ${ }^{22}$ These data suggest the importance of monitoring of serum sodium during administration of linezolid.

\section{Aminoglycosides}

\section{Gentamycin}

It was found that gentamycin affects sodium chloride transport in the thick ascending limb of the loop of Henle. It was found that it increased urine flow, and excretion of sodium, potassium, and chloride after intravenous injection. The glomerular filtration rate and proximal tubule fluid reabsorption were not affected by gentamicin.
In experimental invitro studies, the medullary thick ascending limb was affected by intra-luminal, and not basolateral, administration of gentamicin. These data suggest that hyponatremia induced by gentamycin is a consequence of decreased transport in the thick ascending limb of Henle's loop. ${ }^{23}$

\section{Beta lactam and beta lactamase containing antibiotics}

\section{Cefoperazone/sulbactam}

Beta lactam antibiotics is by far one of the comments antibiotics use in the practice of medicine, particularly the cephalosporin group. Cefoperazone is a $3^{\text {rd }}$ generation cephalosporin that has a wide gram positive and negative coverage with a wide area of body distribution along with the added benefit of Pseudomonas coverage. With the addition of a Beta-lactamase inhibitor such as Sulbactam, the efficacy Cefoperazone rises further. While there were almost no reported cases of an isolated Cephalosporin agent that induced Hyponatremia, this combined drug had a different picture. ${ }^{24}$ The mechanism described behind this is believed to be related to an inappropriate antidiuretic hormone (ADH) activity.

\section{Macrolides}

\section{Azithromycin}

With its powerful gram positive and atypical coverage, Azithromycin is a macrolides antibiotic that is commonly used in variety of situation including respiratory tract infections, sexually transmitted disease, and sometimes in malaria infections. Similar to various type of antibiotics inducing SIADH secretion, an incidence of Azithromycin causing symptomatic hyponatremia was reported in a case report back in the late $1990 \mathrm{~s} .{ }^{25}$ When the patient developed disturbance in her mental status after taking 2 days of Azithromycin therapy, only to be found to have a delusional hyponatremia compatible with a picture of SIADH.

\section{Rifabutin}

Rifabutin is an RNA polymerase inhibiter that is used in tuberculous and non-tuberculous Mycobacterium infection, particularly when Rifampicin cannot be used either due to its side effect, or due to interaction with other medications. A reported HIV patient with mycobacterium infection was started on Rifabutin only to be stopped soon after due to Leukopenia. After re-challenging the patient with another trial of Rifabutin, another episode of leukopenia occurred with new onset picture of delusional hyponatremia. After only discontinuing Rifabutin and following the patient within a week, his leukopenia and hyponatremia started to improve. ${ }^{26}$

\section{Conclusion}

Decreased serum sodium concentration is a rather common finding in the elderly population because of the presence of factors contributing to increased $\mathrm{ADH}$ and the frequent prescription of drugs associated with hyponatremia, such as thiazides or antidepressants. Both the evaluation and the treatment of hyponatremia pose many challenges in the elderly population. Pay special attention when prescribing antimicrobials to the elderly to avoid worsening their sodium level, and to avoid catastrophic attacks of severe hyponatremia.

\section{Acknowledgments}

Authors would like to thank Dr. Samar Badradin, consultant of infectious diseases, the Director of the infection control department at 
Dr. Soliman Fakeeh Hospital (DSFH). She is also the Chief of medical $\&$ clinical Division at DSFH for her kind review of the manuscript and valuable guidance.

\section{Funding details}

None.

\section{Conflicts of interest}

The author declares there is no conflict of interest.

\section{References}

1. Shah. Reappraisal of the risk/benefit of nitrofurantoin: review of toxicity and efficacy. Adverse Drug Reactions and Acute Poisoning Reviews. 1989;8(4):183-201.

2. Clinical and Laboratory Standards Institute. Performance Standards for Antimicrobial Susceptibility Testing; Nineteenth Informational Supplement. CLSI document M100-S19 [ISBN 1-56238-716-2]. Clinical and Laboratory Standards Institute, 940 West Valley Road, Suite 1400, Wayne, Pennsylvania 19087-1898 USA, 2010. p. 1-25.

3. McCalla DR, Kaiser C, Green MH. Genetics of nitrofurazone resistance in Escherichia coli. J Bacteriol. 1978;133(1):10-16.

4. Oplinger M, Andrews CO. Nitrofurantoin contraindication in patients with a creatinine clearance below $60 \mathrm{~mL} / \mathrm{min}$ : looking for the evidence. Ann Pharmacother. 2013;47(1): 106-11.

5. Fick DM, Semla TP, Steinman M, et al. American Geriatrics Society 2019 Updated AGS Beers Criteria for Potentially Inappropriate Medication Use in Older Adults. J Am Geriatr Soc. 2019;67(4):674-694.

6. Mori H, Kuroda Y, Imamura S, et al. Hyponatremia and/or hyperkalemia in patients treated with the standard dose of trimethoprim/ sulfamethoxazole. Intern Med. 2003;42(8):665-669.

7. Kaufman AM, Hellman G, Abramson RG. Renal salt wasting and metabolic acidosis with trimethoprim-sulfamethoxazole therapy. $\mathrm{Mt}$ Sinai J Med. 1983;50(3):238-239.

8. Ahn YH, Goldman JM. Trimethoprim-sulfamethoxazole and hyponatremia. Ann Intern Med. 1985;103(1):161-162.

9. Babayev R, Terner S, Chandra S, et al. Trimethoprim-associated hyponatremia. Am J Kidney Dis. 2013;62(6):1188-1192.

10. Eastel R, Edmonds CJ. Hyponatremia associated with trimethoprim and a diuretic. BMJ. 1984;289(6459):1658-1659.

11. Redding JM, Nichols S, Hallen S. Rapid onset hyponatremia after initiating TMP/SMX and citalopram. J Am Geriatr Soc. 2012;60(Suppl 4):S189-S190.
12. Dunn RL, Smith WJ, Stratton MA. Trimethoprim-sulfamethoxazoleinduced hyponatremia. Consult Pharm. 2011;26(5):342-349.

13. Naranjo CA, Busto U, Sellers EM, et al. A method for estimating the probability of adverse drug reactions. Clin Pharmacol Ther. 1981;30(2):239-245.

14. Babar SM. SIADH associated with ciprofloxacin. Ann Pharmacother. 2013;47(10):1359-1363.

15. Mihaela Mocan, Sorin Nicu Blaga. Severe Hyponatremia due to Levofloxacin Treatment for Pseudomonas aeruginosa CommunityAcquired Pneumonia in a Patient with Oropharyngeal Cancer. Case Rep Med. 2016;2016:5434230.

16. Bolon MK. The newer fluoroquinolones. Infect Dis Clin North Am. 2009;23(4):1027-1051.

17. Liu HH. Safety profile of the fluoroquinolones: focus on levofloxacin. Drug Saf. 2010;33(5):353-369.

18. Kushner JM, Peckman HJ, Snyder CR. Seizures associated with fluoroquinolones. Ann Pharmacother. 2001;35(10):1194-1198.

19. Mussig K, Schnauder G, Morike K. Severe and symptomatic hyponatraemia after moxifloxacin intake. Neth J Med. 2009;67:197.

20. Yam FK, Eraly SA. Syndrome of inappropriate antidiuretic hormone associated with moxifloxacin. Am J Health Syst Pharm. 2012;69(3):217220 .

21. Tanaka R, Suzuki Y, Takumi Y, et al. A Retrospective Analysis of Risk Factors for Linezolid-Associated Hyponatremia in Japanese Patients. Biol Pharm Bull. 2016;39(12):1968-1973.

22. Ioannou P, Stavroulaki M, Mavrikaki V, et al. A case of severe hyponatremia due to linezolid-induced SIADH. J Clin Pharm Ther. 2018;43(3):434-436.

23. Kidwell DT, McKeown JW, Grider JS, et al. Acute effects of gentamicin on thick ascending limb function in the rat. Eur $J$ Pharmacol. 1994;270(1):97-103.

24. Basu S, Mitra S. Cefoperazone / sulbactam induced hyponatremia. Indian J Med Sci. 2006;60(4):158-159.

25. Cadle RM, Darouiche RO, Ashton CM. Symptomatic Syndrome of Inappropriate Antidiuretic Hormone Secretion Associated with Azithromycin. Ann Pharmacother. 1997;31(11):1308-1310.

26. Chitre MM, Berenson CS. Idiosyncratic Rifabutin-Induced Leukopenia and SIADH: Case Report and Review. Pharmacotherapy. 2001;21(4):493-497. 